\title{
A high-performance silicon-on-insulator MEMS gyroscope operating at atmospheric pressure
}

\author{
Said Emre Alper*, Kivanc Azgin, Tayfun Akin \\ Middle East Technical University, Department of Electrical and Electronics Eng., Ankara, Turkey \\ Received 28 February 2006; received in revised form 9 June 2006; accepted 13 June 2006 \\ Available online 27 July 2006
}

\begin{abstract}
This paper presents a new, high-performance silicon-on-insulator (SOI) MEMS gyroscope with decoupled oscillation modes. The gyroscope structure allows it to achieve matched-resonance-frequencies, large drive-mode oscillation amplitude, high sense-mode quality factor, and low mechanical cross-talk. The gyroscope is fabricated through the commercially available SOIMUMPS process of MEMSCAP Inc. The fabricated gyroscope has minimum capacitive sense gaps of $2.6 \mu \mathrm{m}$ and a structural silicon thickness of $25 \mu \mathrm{m}$, and it fits into a chip area smaller than $3 \mathrm{~mm} \times 3 \mathrm{~mm}$. The fabricated gyroscope is hybrid connected to a CMOS capacitive interface ASIC chip, which is fabricated in a standard $0.6 \mu \mathrm{m}$ CMOS process. The characterization of the hybrid-connected gyroscope demonstrates a low measured noise-equivalent rate of $90^{\circ} / \mathrm{h} / \mathrm{Hz}^{1 / 2}$ at atmospheric pressure, eliminating the need for a vacuum package for a number of applications. $R^{2}$-non-linearity of the gyroscope is measured to be better than $0.02 \%$. The gyroscope has a low quadrature signal of $70 \% \mathrm{~s}$ and a short-term bias stability of $1.5 \%$ s. The angular rate sensitivity of the gyroscope is $100 \mu \mathrm{V} /(\% / \mathrm{s})$ at atmospheric pressure, which improves 24 times to $2.4 \mathrm{mV} /(\% / \mathrm{s})$ at vacuum. The noise-equivalent rate of the gyroscope at $20 \mathrm{mTorr}$ vacuum is measured to be $35^{\circ} / \mathrm{h} / \mathrm{Hz}^{1 / 2}$, which can be improved further by reducing the electromechanical noise.
\end{abstract}

(C) 2006 Elsevier B.V. All rights reserved.

Keywords: Gyroscope; Angular rate sensor; SOI gyroscope; Mechanical decoupling

\section{Introduction}

The need for high-performance micromachined gyroscopes force the research towards dedicated mechanical designs combined with improved microfabrication technologies and highsensitivity readout and control electronics. Among many micromachined gyroscopes, Coriolis vibratory rate gyroscopes have demonstrated significant progress within the past decade satisfying the requirements of several applications, such as guidance, robotics, tactical-grade navigation, and automotive applications [1-4]. The performance of Coriolis rate gyroscopes can be boosted further by implementing symmetric and decoupled structures that allow easy mode-matching, while reducing the mechanical cross-talk between the drive- and sense-modes of the gyroscope. Currently, such structures are reported with completely symmetric mode shapes and mechanically decoupled suspensions [5-8]. The absolute symmetry in these designs

\footnotetext{
* Corresponding author. Tel.: +90 3122104415 ; fax: +90 3122102304 .

E-mail address: said@metu.edu.tr (S.E. Alper).
}

requires linear comb-drives for both the drive- and sense-modes; however, linear comb-drives prevent effective post-process tuning of the resonance frequencies and reduce the mechanical sensitivity of the gyroscope. In addition, the previous symmetric designs use clamped-guided type suspension beams that limit the drive-mode vibration amplitude to less than few microns, preventing higher sensitivity. Furthermore, most of the symmetric and decoupled gyroscopes in literature require vacuum packaging, increasing the unit cost and reducing the number of eligible application platforms. Therefore, there is a need to develop a new mechanical gyroscope structure that can overcome the limitations of previous symmetric and decoupled gyroscopes [5-8], while keeping their advantages, so that the gyroscopes can provide high-performance even at atmospheric pressure, which is very important for reducing the packaging cost and complexity.

This paper reports a new symmetric and decoupled silicon microgyroscope structure [9] that has (1) varying-gap type sense electrodes for improved rate sensitivity, (2) post-process electrostatic frequency tuning capability, and (3) symmetric and folded suspensions for mechanical decoupling and large drive-mode 
vibration amplitudes. The structure is fabricated using a commercially available high-aspect-ratio silicon-on-insulator (SOI) micromachining process that allows high mechanical quality factors. The mature fabrication technology together with the improvements of the electromechanical design the gyroscope demonstrates a high-performance even at atmospheric pressure, eliminating the need for vacuum packaging for a number of applications.

\section{Electromechanical design}

Fig. 1 shows the perspective view of the new symmetric and decoupled gyroscope. The drive-mode of the gyroscope is optimized for large amplitude and linear driving oscillations, whereas the sense-mode is optimized for increased rate sensitivity and electrostatic frequency tuning. Suspension flexures are designed symmetric along the drive- and sense-modes for minimizing possible temperature-dependent drift. Undesired mechanical cross-talk between the drive- and sense-modes is minimized by proper design of suspension flexures and anchorage of the structure, restricting the motion of the movable drive and sense electrodes to one degree-of-freedom (d.f.) and allowing the two d.f. motion only for the proof mass element. In addition, the vibrating mass is mechanically isolated from the sense jigs by carefully locating the flexures so that the moments and forces created on the sense jigs cancels the disturbing torques acting on electrodes during linear drive-mode vibrations. The advantages of the new structure include large sense capacitances, large drive-mode vibration amplitude, and electrostatic frequency tuning capability, while the symmetry of the suspension beams and mechanical decoupling of the driveand sense-modes are still preserved as in [6-8]. These features and improvements yield remarkable performance when combined with the advantages of a standard SOI-MEMS foundry process.

Fig. 2 shows the mode shapes of the designed SOI gyroscope for (a) drive and (b) sense-modes, simulated using the Coventor-

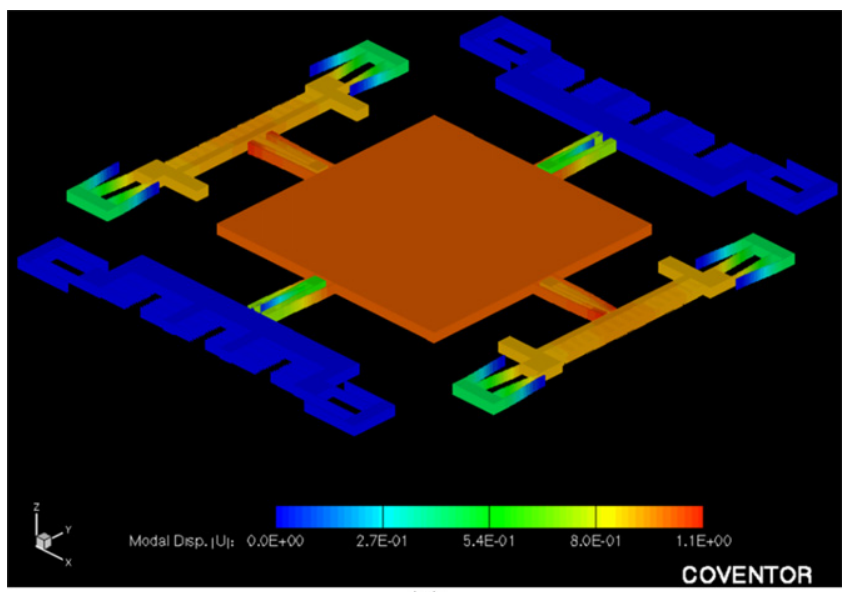

(a)

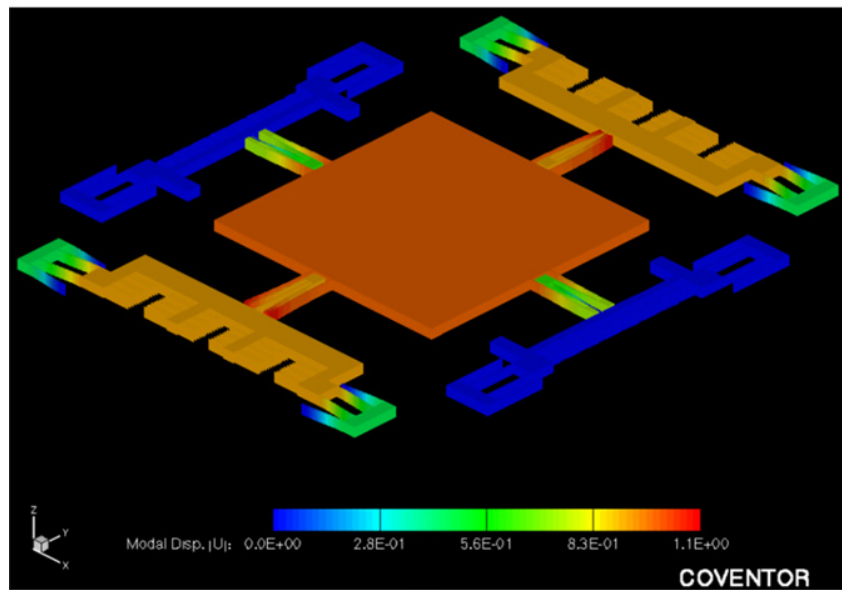

(b)

Fig. 2. Mode shapes of the designed SOI gyroscope for (a) drive and (b) sensemodes, simulated using CoventorWare finite-element solver

Ware finite-element solver. Clearly, the interaction between the drive and sense electrodes of the SOI gyroscope is highly suppressed as the sense electrode remains almost stationary during the deflection of the drive-mode.

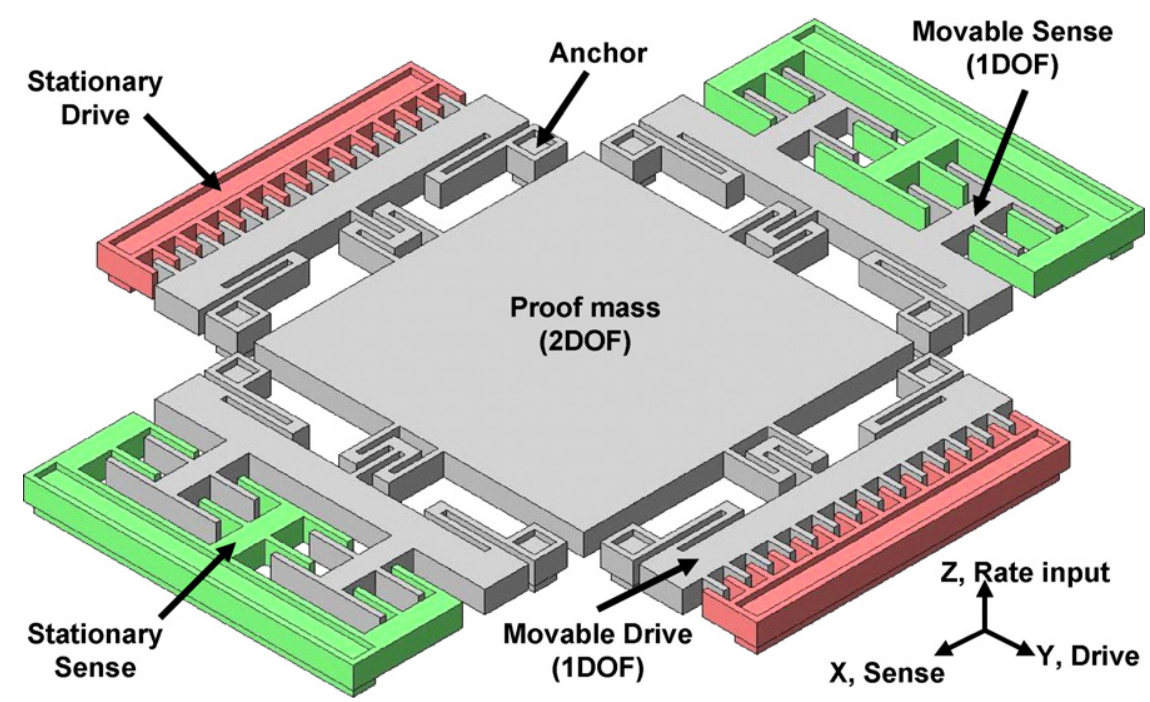

Fig. 1. Perspective view of the improved symmetrical and decoupled gyroscope structure. 


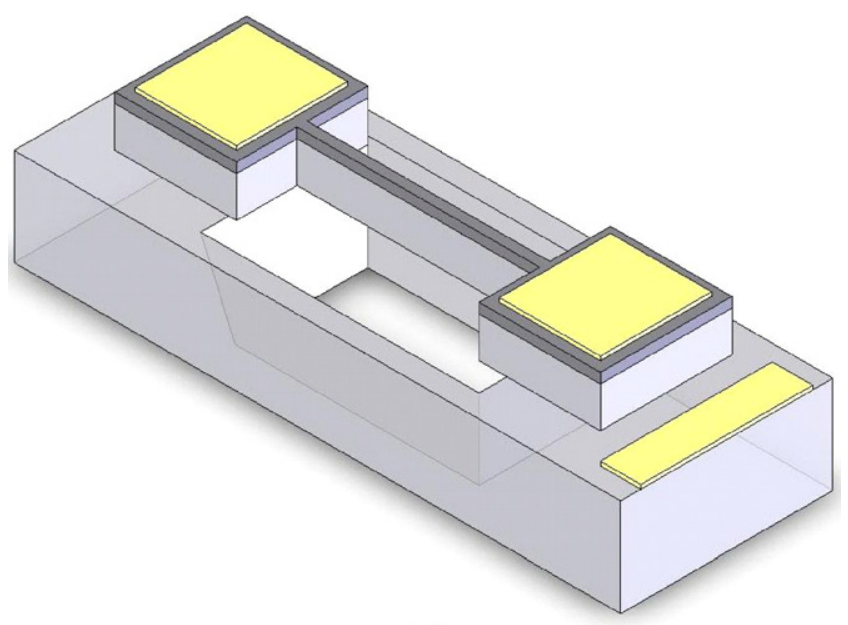

(a)

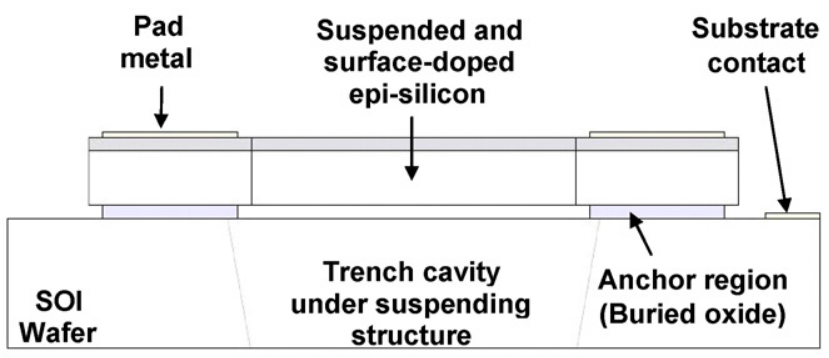

(b)

Fig. 3. (a and b) Typical structure and cross-section of a cantilever structure fabricated through SOIMUMPS. The portion of the handle wafer underneath the suspending structures is totally removed by DRIE from the backside of the handle wafer.

\section{Fabrication process}

The gyroscope is fabricated using the standard SOIMUMPS process of MEMSCAP Inc. [10]. The process includes frontside and backside patterning of an SOI wafer using deep-reactive ion etch (DRIE). The detailed process steps are not given here, as it can be obtained at [10]. Fig. 3 shows the typical structure and cross-section of a cantilever structure fabricated through the SOIMUMPS process, where the structural layer thickness can be either 10 or $25 \mu \mathrm{m}$. The portion of the handle wafer underneath the suspending structures is totally removed by DRIE from the backside of the handle wafer. The thorough etching of the substrate under the suspended structures significantly reduces the air damping and yields high mechanical quality factors. Another advantage of removing the substrate layer under the suspended structures is that high dc potentials can safely be applied to the movable structures, as the effects of electrostatic levitation are almost completely eliminated.

\section{Characterization results}

Fig. 4 shows the SEM pictures of the fabricated gyroscope, which has a $25 \mu \mathrm{m}$-thick silicon structural layer. The capacitive gaps are measured to be as large as $2.6 \mu \mathrm{m}$, although they were drawn to be $2 \mu \mathrm{m}$ in the layout. Table 1 presents a comparison of the designed, measured, and estimated electrical and mechanical parameters for the fabricated SOI-MEMS gyroscope. In general, the designed values agree well with the measured values considering that the capacitive gap spacing and flexure beam widths are slightly different than the design values due to fabrication tolerances. The drive and sense electrode capacitances of the gyroscope are measured using Agilent 4294A precision impedance analyzer as 991 and $1261 \mathrm{fF}$, respectively. These values agree well with the estimated values of 863 and $1378 \mathrm{fF}$, respectively, considering $2.6 \mu \mathrm{m}$ capacitive gaps. The parasitic capacitances associated with the drive and sense electrodes are in a close range about $3 \mathrm{pF}$, typical for any sensor built on an SOI substrate.

Fig. 5 shows the measurements of drive- and sense-mode resonance characteristics for the fabricated gyroscope using the Agilent 4395A network analyzer and Karl Suss micromanipulator probe station. The resonance frequencies of the drive- and the sense-modes of the fabricated gyroscope are measured to be matched at about $5.3 \mathrm{kHz}$ for a dc polarization voltage of $8.4 \mathrm{~V}$ is applied to the proof mass. Both the measured drive- and sense-mode mechanical resonance frequencies are close to the expected values of $5.42 \mathrm{kHz}$. The voltage required to match the mode frequencies is somewhat higher than the design value of

Table 1

Comparison of the designed, measured, and estimated electrical and mechanical parameters for the fabricated SOI gyroscope

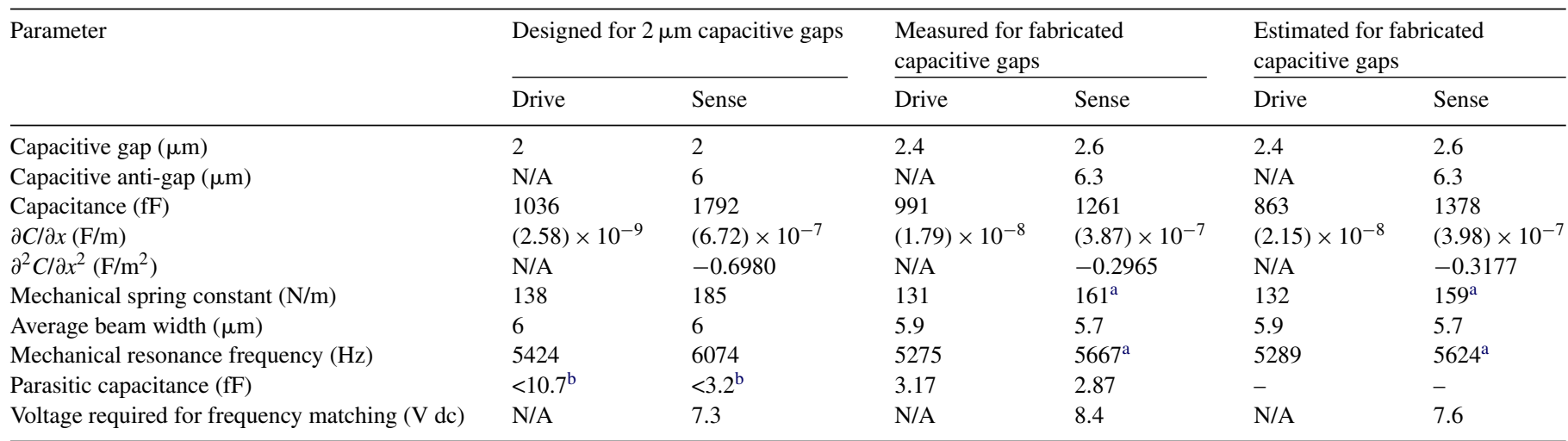

\footnotetext{
a Includes only the extracted mechanical spring constant.

b Anchor size for the drive electrode is larger than that of the sense electrode.
} 

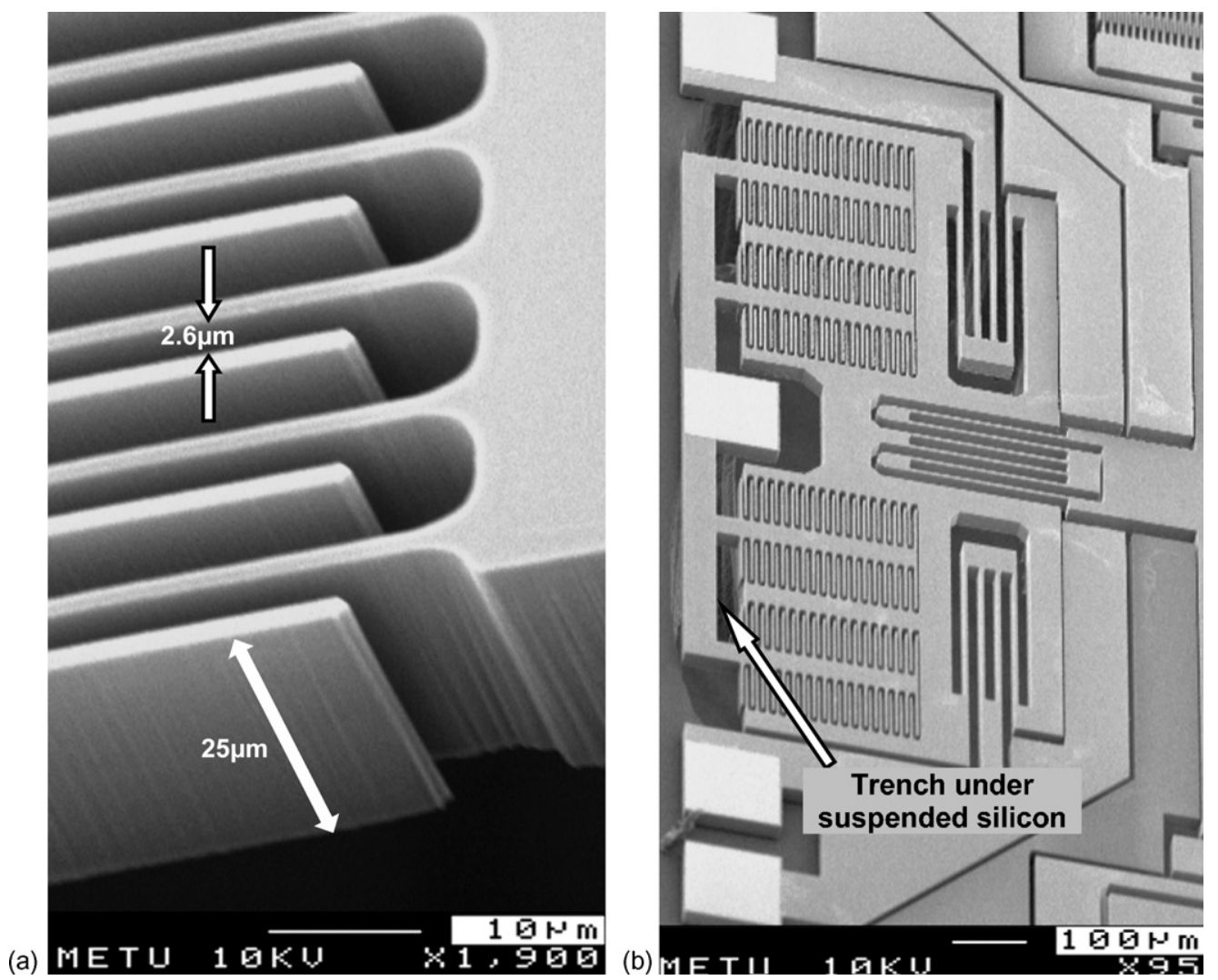

Fig. 4. SEM pictures of the fabricated gyroscope, showing (a) $25 \mu \mathrm{m}$-thick comb fingers with $2.6 \mu \mathrm{m}$ capacitive gap, and (b) suspended sense-electrode of the fabricated gyroscope.

$7.3 \mathrm{~V}$, simply because of the capacitive gaps enlarge from $2 \mu \mathrm{m}$ design values to $2.6 \mu \mathrm{m}$ during fabrication process reducing the effective electrostatic spring constant associated with the sensemode.

The quality factors of the SOI gyroscope for the drive- and the sense-modes at atmospheric pressure are then measured to be 462 and 11, respectively. The lower quality factor of the sensemode is a result of higher air damping along the sense-mode due to the high-aspect-ratio varying-gap type sense electrodes. Still, the removal of the substrate portion under the vibrating mass prevents a further reduction of the quality factors and yield significant performance even at atmospheric pressure, as discussed next.

The fabricated gyroscope is hybrid connected to a singleended CMOS capacitive interface circuit [7]. Fig. 6 shows the schematic view of the CMOS capacitive interface circuit. The capacitive interface circuit has high input-impedance in order to buffer the high-impedance sensor output. High input impedance is achieved by minimizing the parasitic capacitance associated with the CMOS chip using bootstrapping method, whereas the parasitic capacitance associated with the gyroscope chip is already minimized due to insulating substrate. The designed capacitive interface circuit is fabricated in XFAB $0.6 \mu \mathrm{m}$ CMOS process and measures only $0.7 \mathrm{~mm} \times 1.3 \mathrm{~mm}$. Fig. 7 shows the photograph of the fabricated SOI-MEMS gyroscope hybrid connected to the CMOS capacitive interface circuit inside a 40-pin DIL package.
Fig. 8 demonstrates the effective frequency tuning for the sense-mode of the SOI-MEMS gyroscope. The sensemode resonance frequency of the gyroscope can be reduced from $5.55 \mathrm{kHz}$ at $5 \mathrm{~V} \mathrm{dc}$ down to $4.72 \mathrm{kHz}$ at $13 \mathrm{~V}$ dc by a negative electrostatic spring constant. The total electrostatic spring constant of the fabricated gyroscope is found to be $-(0.2965) V^{2}$, where $V$ is the dc voltage across the varyinggap type sense electrodes. The measured electrostatic spring constant is in close agreement with the theoretically expected value of $-(0.3177) V^{2}$ calculated for $2.6 \mu \mathrm{m}$ capacitive gap spacing.

The fabricated gyroscope is excited into self-resonance along the drive-mode at atmospheric pressure using an electronic positive-feedback loop. The estimated vibration amplitude of the gyroscope is about $18.8 \mu \mathrm{m}$ without introducing a significant non-linearity, owing to its stress-relieving suspensions. Next, the rate sensitivity of the gyroscope is measured using a single-axis rate table. Fig. 9 shows the measured sense-mode raw output of the SOI-MEMS gyroscope prior to demodulation, in response to a sinusoidal angular rate input with an amplitude of $2 \pi^{\circ} / \mathrm{s}$ and having a frequency of $10 \mathrm{~Hz}$. At atmospheric pressure, the mechanical rate sensitivity and total RMS electromechanical noise floor are measured as $100 \mu \mathrm{Vpeak} /\left({ }^{\circ} / \mathrm{s}\right)$ and $2.5 \mu \mathrm{V} / \mathrm{Hz}^{1 / 2}$, respectively. The noise-equivalent rate of the gyroscope then corresponds to $90^{\circ} / \mathrm{h}$ in $1 \mathrm{~Hz}$ measurement bandwidth, which is a remarkable performance for operation at atmospheric pressure. The measured quadrature signal at the sense-mode output 

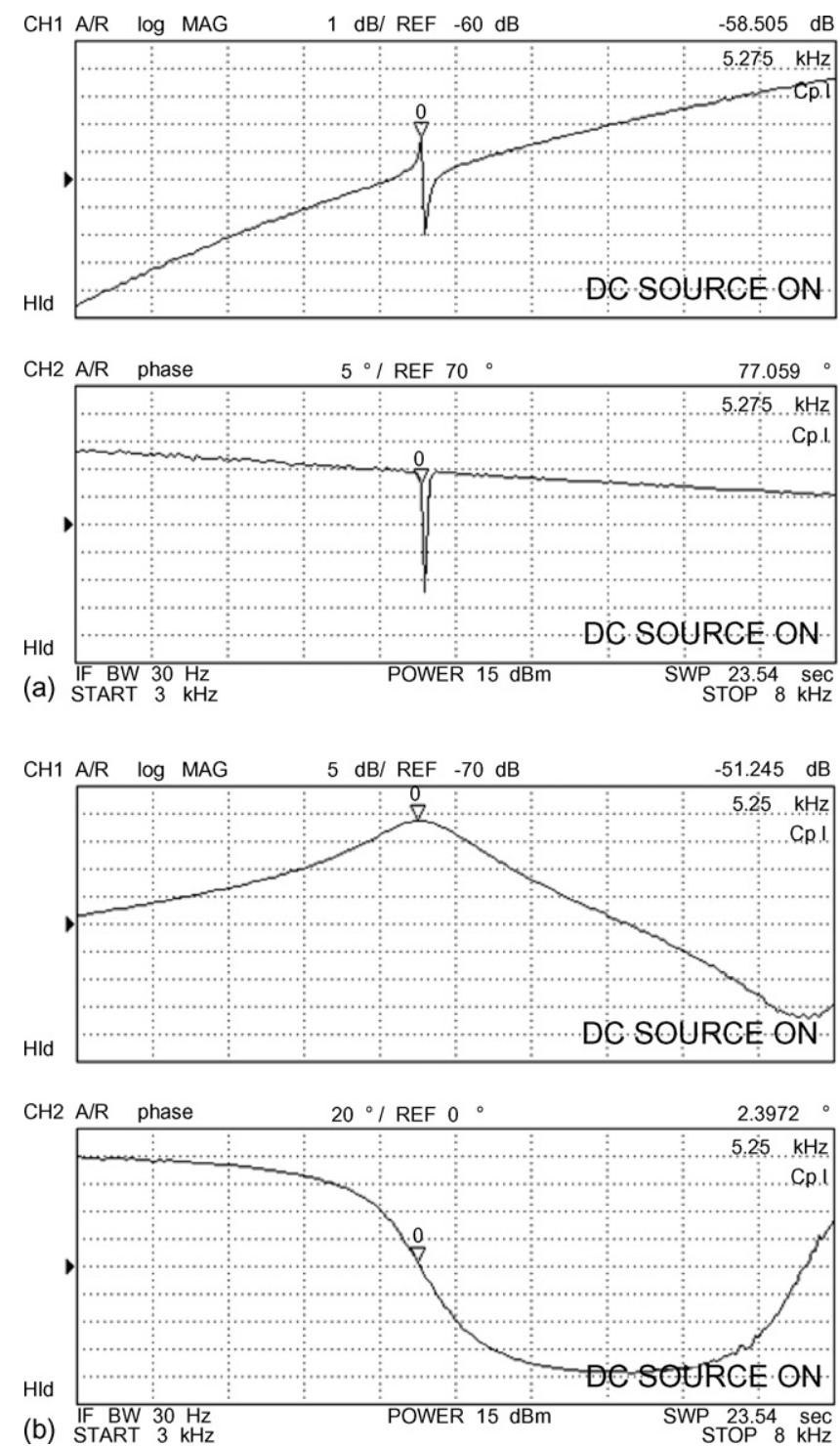

Fig. 5. Measurements of (a) drive and (b) sense-mode resonance characteristics for the fabricated gyroscope using Agilent 4395A network analyzer and Karl Suss micromanipulator probe station.

is less than $70^{\circ} \mathrm{s}$ without applying any balancing scheme for quadrature cancellation, owing to the fully decoupled structure of the gyroscope.

The raw output of the gyroscope shown in Fig. 9 is then demodulated by a carrier sampled from the output of the selfresonating drive-mode of the gyroscope, and the resulting signal is filtered through a simple first-order low-pass filter, providing a dc output voltage proportional to the applied angular rate input. It should be noted that the circuit blocks other than the CMOS capacitive interface circuit are all constructed on a printedcircuit-board (PCB), using off-the-shelf IC multiplier, op amps, and passive components. Fig. 10 shows the measured dc output of the gyroscope in response to constant angular rate inputs from zero-rate up to $\pm 100 \%$ s using $10 \%$ s steps and then back to zero-rate. Clearly, the gyroscope yields a repeatable response in each measurement step and turns back to its original zero-rate state without a significant hysteresis.

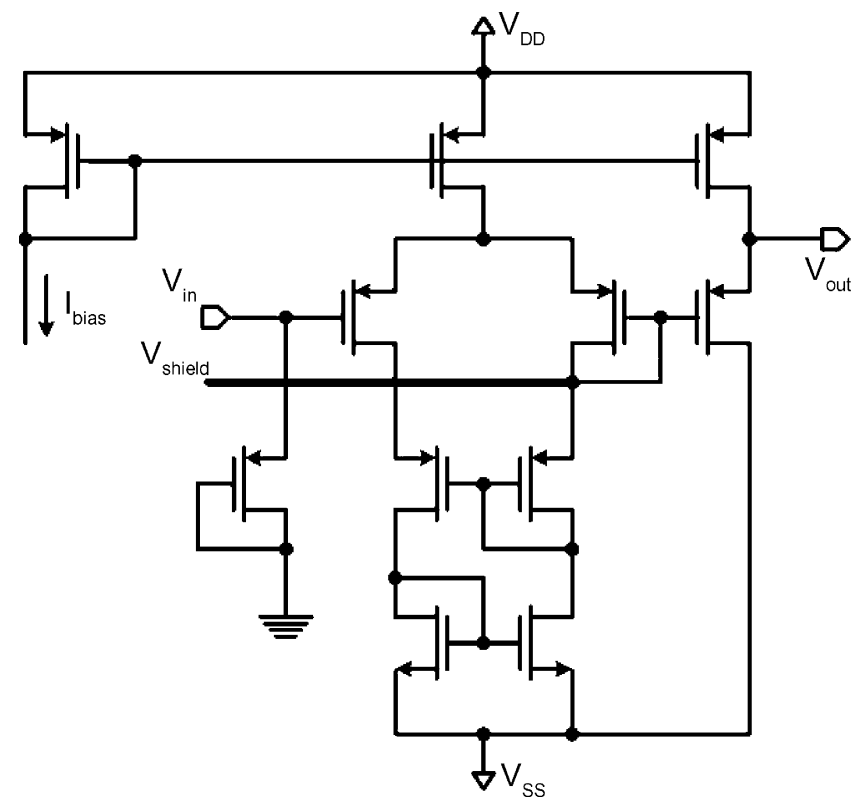

Fig. 6. Schematic view of the CMOS capacitive interface circuit.

Fig. 11 shows the input rate versus the output dc voltage characteristics of the gyroscope. The gyroscope demonstrates a scale factor of $15.2 \mathrm{mV} /(\% / \mathrm{s})$ in a measurement range of $\pm 100^{\circ} / \mathrm{s}$, with $40 \mathrm{~dB}$ amplification of the raw sense output. The zero-rate offset of the output is less than $20^{\circ}$ /s, which is limited by the demodulation phase error, arising due to the use of a low-cost analog multiplier as a demodulator and due to the signal routing on PCB. Using a balanced demodulator and improving signal routing would yield the offset below $1 \%$ s. The $R^{2}$-non-linearity of the measured scale factor is better than $0.02 \%$ within $\pm 100^{\circ} / \mathrm{s}$ full scale. The remarkable linearity of the output is basically due to the stress relieving flexures that provide linear deflections.

Fig. 12 shows the measured random variation of the output bias of the fabricated SOI-MEMS gyroscope for a zero-rate input. Bias stability of the fabricated gyroscope is above $1 \%$ for short-term (100 s) navigation, which is dominated by the phaseerror and the drift of the low-cost demodulating electronics. The fluctuations in the dc bias of the SOI handle wafer also affect the overall bias stability, which can be minimized by using sufficient number of optimally located substrate contacts in future designs. The overall bias stability of the gyroscope is better than $7 \%$ for long-term $(3 \mathrm{~h})$ measurements.

The performance of the gyroscope is also evaluated at 20 mTorr vacuum ambient, using an amplitude-controlled drivemode resonance vibration amplitude of $18.9 \mu \mathrm{m}$. Fig. 13 shows the measured sense-mode raw output of the gyroscope at 20 mTorr vacuum ambient, in response to a sinusoidal angular rate input with an amplitude of $2 \pi^{\circ} / \mathrm{s}$ and a frequency of $10 \mathrm{~Hz}$. The rate sensitivity of the gyroscope improves to above $2.4 \mathrm{mVpeak} /(\% / \mathrm{s})$, however, the RMS noise-floor also increases to $22.4 \mu \mathrm{V} / \mathrm{Hz}^{1 / 2}$, due to increased mechanical crosstalk between the drive- and sense-modes at vacuum. These measured values correspond to a noise-equivalent rate of $35^{\circ} / \mathrm{h}$ in $1 \mathrm{~Hz}$ measurement bandwidth. The increase in the RMS noise-floor due to the mechanical cross-talk at vacuum lim- 


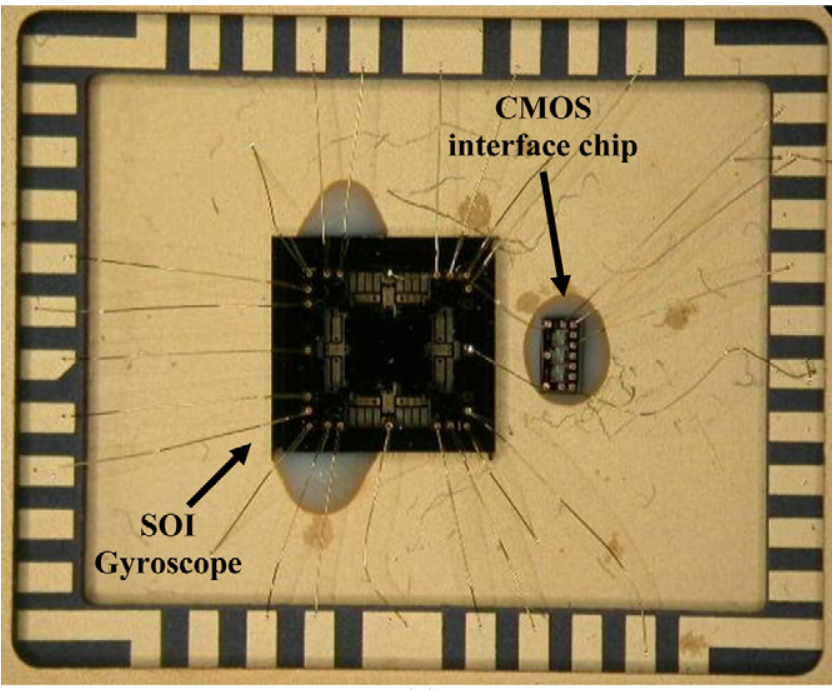

(a)

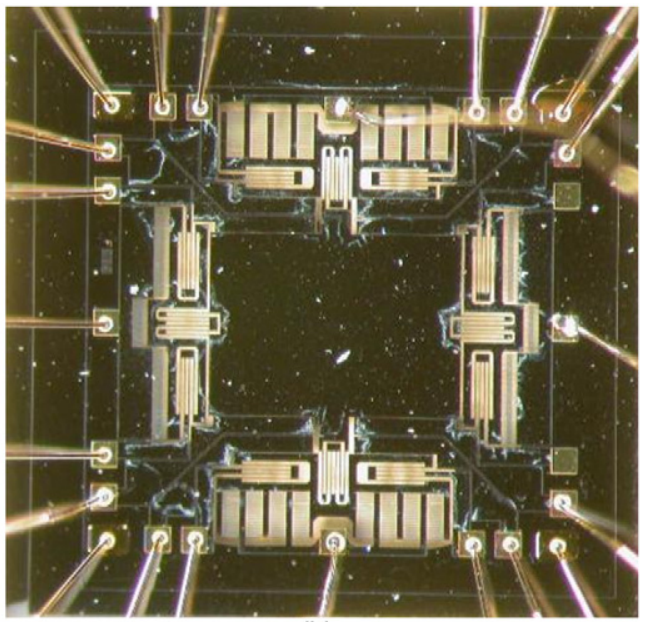

(b)

Fig. 7. (a) Photograph of the fabricated SOI gyroscope hybrid connected to the CMOS capacitive interface circuit inside a 40-pin DIL package. (b) Close-up view of the gyroscope showing the wirebonds to the pads of the CMOS chip and package.

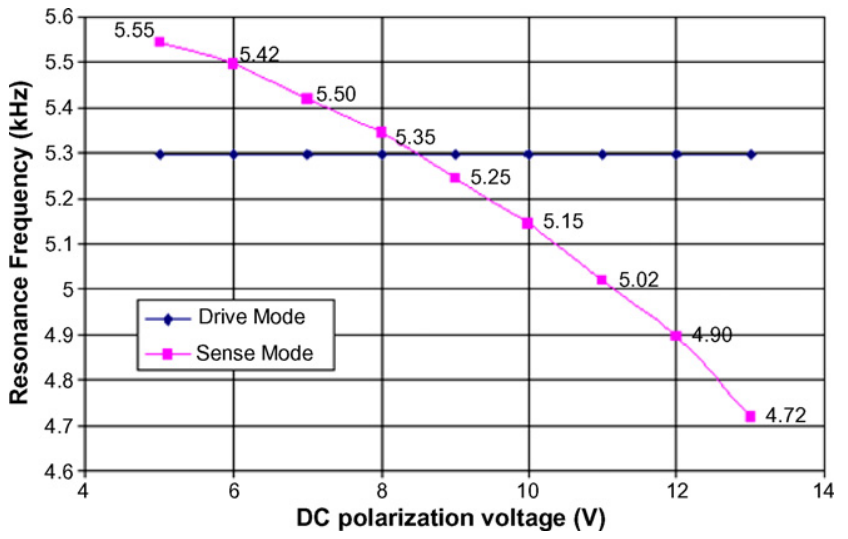

Fig. 8. Demonstration of effective frequency tuning for the sense-mode of the SOI gyroscope. The sense-mode resonance frequency of the gyroscope can be reduced from $5.55 \mathrm{kHz}$ at $5 \mathrm{~V}$ dc down to $4.72 \mathrm{kHz}$ at $13 \mathrm{~V}$ dc by negative electrostatic spring constant.

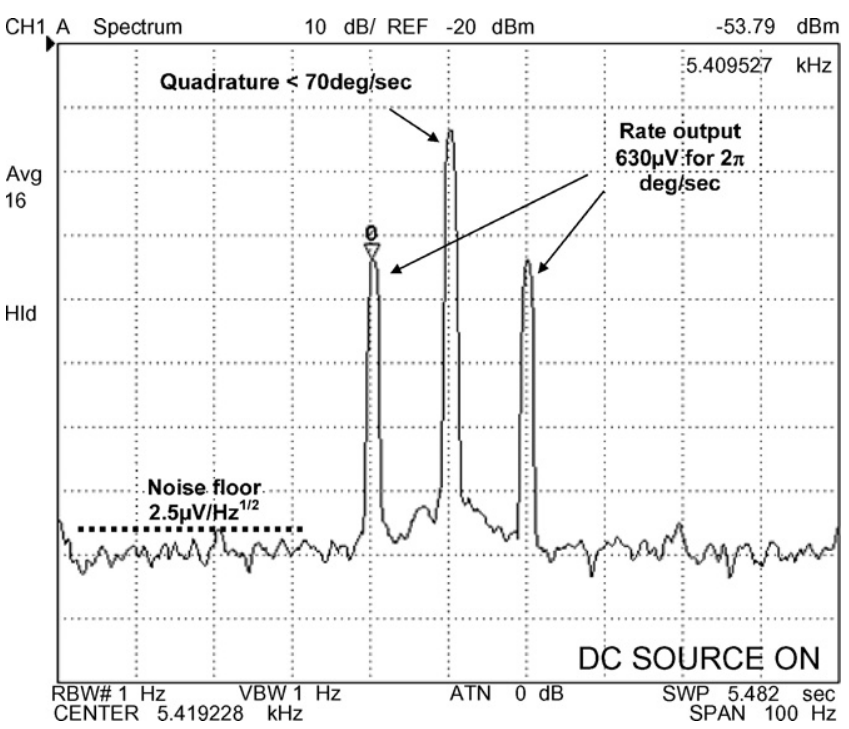

Fig. 9. Measured sense-mode raw output of the SOI-MEMS gyroscope prior to demodulation, in response to a sinusoidal angular rate input with an amplitude of $2 \pi^{\circ} / \mathrm{s}$ and having a frequency of $10 \mathrm{~Hz}$.

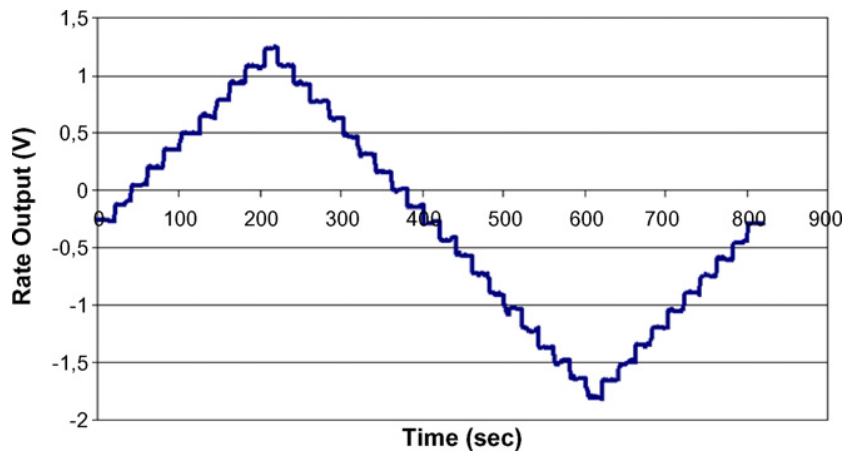

Fig. 10. Measured dc output of the gyroscope in response to constant angular rate inputs from zero-rate up to $\pm 100^{\circ} / \mathrm{s}$ using $10^{\circ} / \mathrm{s}$ steps and then back to zero-rate.

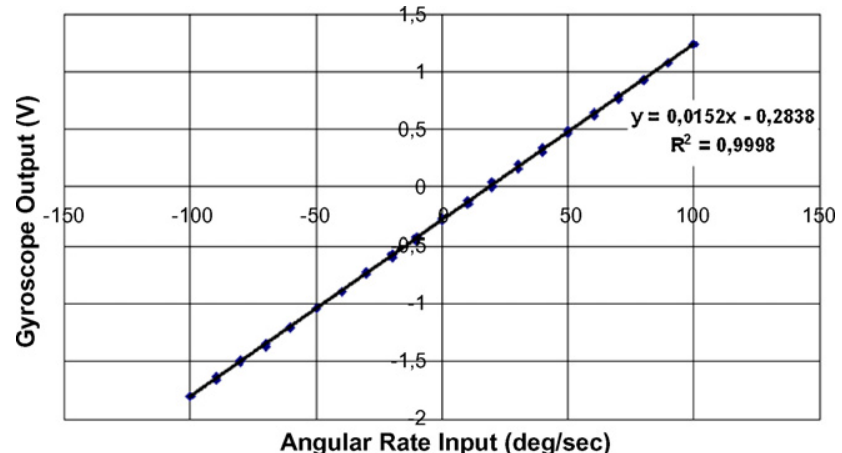

Fig. 11. Measured scale factor and $R^{2}$-non-linearity of the output response of the gyroscope. The gyroscope demonstrates a scale factor of $15.2 \mathrm{mV} /\left({ }^{\circ} / \mathrm{s}\right)$ in a measurement range of $\pm 100 \%$ s, with $40 \mathrm{~dB}$ amplification of the raw sense output. The $R^{2}$-non-linearity of the measured scale factor is better than $0.02 \%$ within $\pm 100^{\circ} / \mathrm{s}$ full scale. 


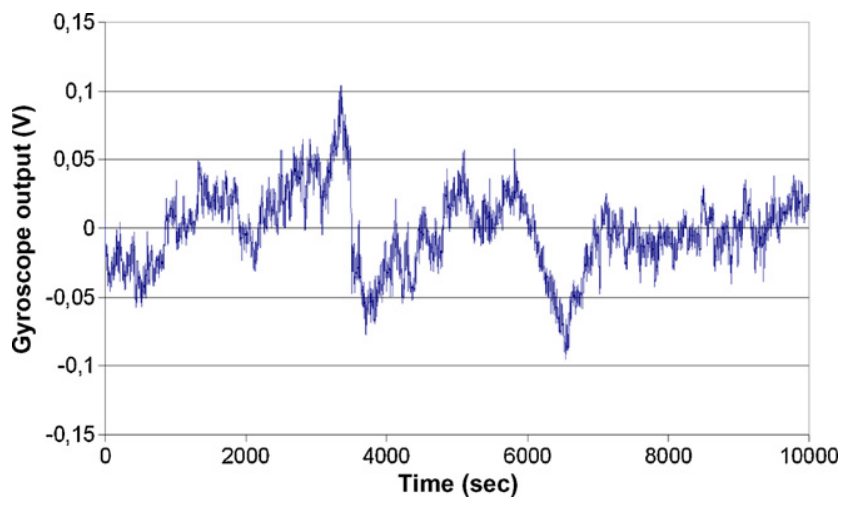

Fig. 12. Measured random variation of the output bias of the fabricated SOI gyroscope for zero-rate input. Bias stability of the fabricated gyroscope is above $1 \%$ for short-term $(100 \mathrm{~s})$ navigation, which is dominated by the phase-error and the drift of the low-cost demodulating electronics.

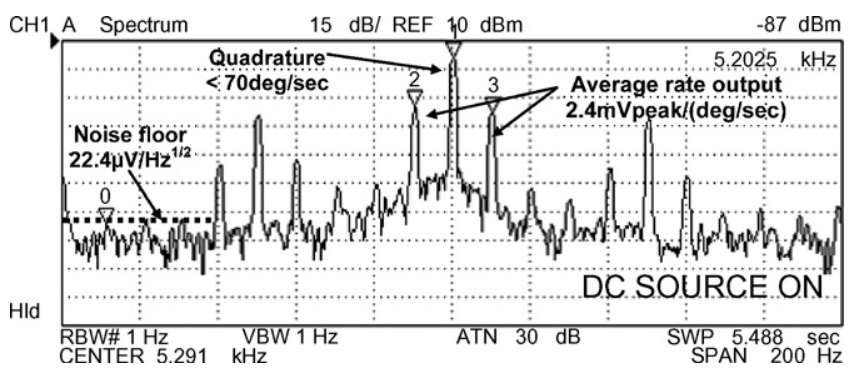

Fig. 13. Measured sense-mode raw output of the gyroscope at 20 mTorr vacuum ambient, in response to a sinusoidal angular rate input with an amplitude of $2 \pi / \mathrm{s}$ and a frequency of $10 \mathrm{~Hz}$. The rate sensitivity of the gyroscope improves to above $2.4 \mathrm{mVpeak} /(\% / \mathrm{s})$, however, the RMS noise-floor also increases to $22.4 \mu \mathrm{V} / \mathrm{Hz}^{1 / 2}$, due to increased mechanical cross-talk between the drive- and sense-modes at vacuum.

its the performance improvement of the fabricated gyroscope during vacuum operation. The increased mechanical cross-talk is believed to be due to the susceptibility of individual sense electrodes to rotational vibrations. The performance at vacuum

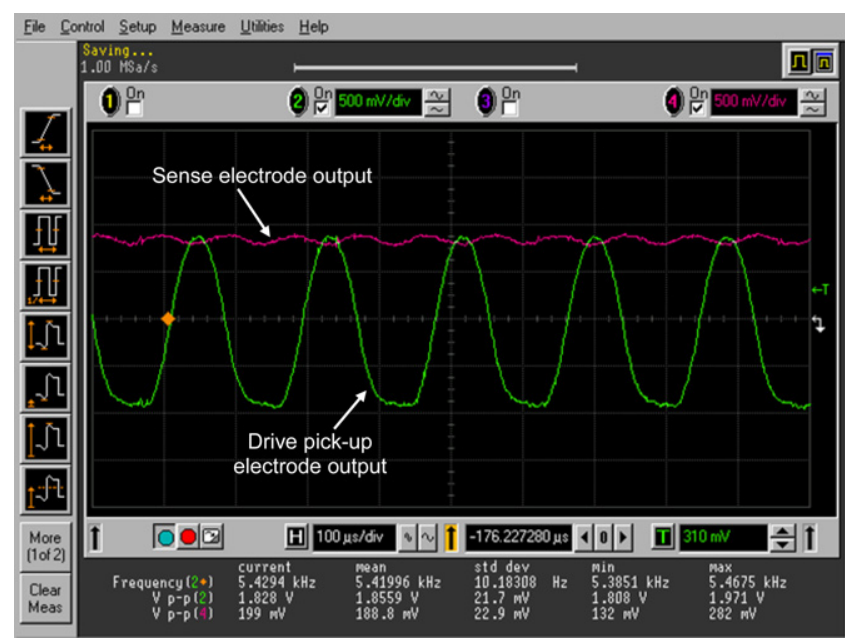

Fig. 14. Measured real-time outputs of the drive and sense electrodes of the gyroscope in the absence of an applied angular rate. Clearly, there exist a timevarying signal at the sense output, and this signal has a frequency twice that of the drive-mode resonance frequency.

can be increased significantly by further reducing the mechanical cross-talk between the drive- and sense-modes with the use of a more-rigid sense-frame, which is considered for future designs.

Fig. 14 shows the measured real-time outputs of the drive and sense electrodes of the gyroscope in the absence of an applied angular rate. Clearly, there exist a time-varying signal at the sense output, and this signal has a frequency twice that of the drive-mode resonance frequency. Fig. 15 describes the source for the double-frequency motion. The sense-mode electrodes of the decoupled micromachined gyroscope structure of Fig. 1 are quasi-stationary during the drive-mode oscillations, i.e., they do not cause any motion along the sense-mode with a frequency identical to the drive-mode frequency. However, the drive-mode

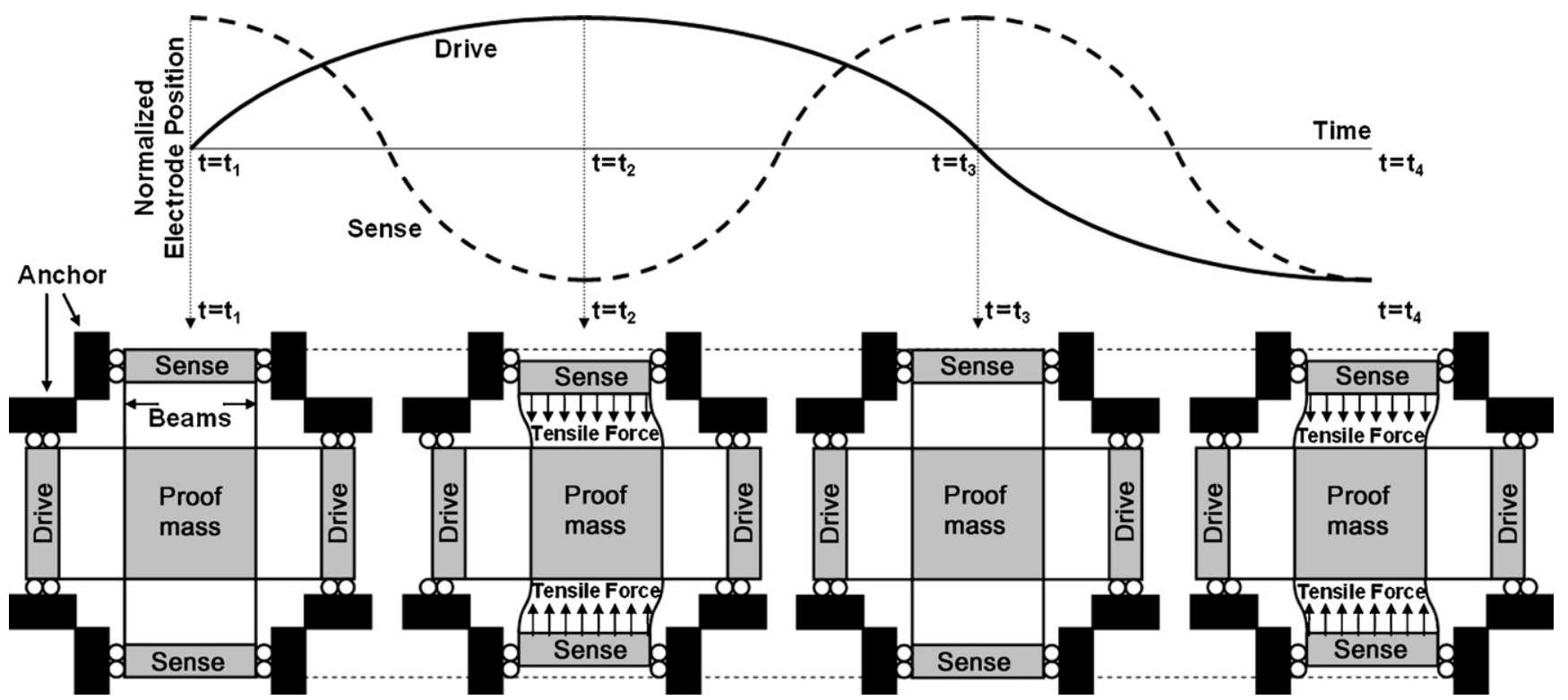

Fig. 15. Source for the frequency-doubling at the sense-mode. The sense-mode electrode is pulled down twice during the completion of a single cycle of drive-mode oscillations. 
oscillations cause another type of motion of the sense electrode with a frequency twice that of the drive-mode oscillations, which arises due to the small but finite axial stresses induced in the flexible beams attached between the sense electrodes and the proof mass. Fortunately, the double-frequency motion causes a common-mode capacitance change at both sense electrodes, which can be suppressed to a great extent by differential sensing scheme. Still, it might be considered in future designs to build a rigid mechanical connection between the differential sense electrodes in order to suppress this double frequency motion, which is another possible source for increased mechanical crosstalk at vacuum.

\section{Conclusions}

This paper reports a new, high-performance SOI-MEMS gyroscope with decoupled oscillation modes, demonstrating a minimum-detectable angular rate of $90^{\circ} / \mathrm{h} / \mathrm{Hz}^{1 / 2}$ at atmospheric pressure. This performance is impressive considering that it is at atmospheric pressure, and it eliminates the need for vacuum packaging of the gyroscope for a number of applications. The gyroscope provides a scale factor non-linearity of $0.02 \%$ within $\pm 100 \%$ s measurement range and has a low quadrature signal of $70 \%$ s. The bias stabilities of the gyroscope are determined to be better than 1.5 and $7 \% \mathrm{~s}$ for short-term $(100 \mathrm{~s})$ and long-term $(3 \mathrm{~h})$ measurements, respectively, dominated by the demodulating electronics and substrate bias fluctuations. The measured performance of the gyroscope at $20 \mathrm{mTorr}$ vacuum is $35^{\circ} / \mathrm{h} / \mathrm{Hz}^{1 / 2}$, which can be further increased by improving the mechanical structure to have a more rigid sense frame in the future designs, by using on-chip balanced demodulators instead of low-cost analog multiplier ICs, and by increasing the number of substrate contacts.

\section{Acknowledgements}

This work is supported by The State Planning Organization (DPT) of Turkey. Authors also appreciate Kanber Mithat Silay for his efforts during the tests of the fabricated gyroscope.

\section{References}

[1] N. Yazdi, F. Ayazi, K. Najafi, Micromachined inertial sensors, Proc. IEEE 86 (August (8)) (1998) 1640-1659.

[2] K. Funk, H. Emmerich, A. Schilp, M. Offenberg, R. Neul, F. Larmer, A surface micromachined silicon gyroscope using a thick polysilicon layer, in: The 12th IEEE Int. Conf. Micro Electro Mechanical Systems Workshop (MEMS'99), Orlando, FL, January 1999, pp. 57 60.

[3] G. He, K. Najafi, A single-crystal silicon vibrating ring gyroscope, in: Proc. 15th IEEE Int. Conf, Las Vegas, CA, January 2002, pp. 718721.

[4] J.A. Geen, S.J. Sherman, J.F. Chang, S.R. Lewis, Single-chip surface micromachined intergated gyroscope with $50^{\circ} / \mathrm{h}$ allan deviation, J. Solid State Circuits 37 (December (12)) (2002) 1860-1866.

[5] M.S. Kranz, G.K. Fedder, Micromechanical vibratory rate gyroscopes fabricated in conventional CMOS, in: Symp. on Gyro Tech., 1997, pp. 3.0-3.8.
[6] S.E. Alper, T. Akin, A symmetric surface micromachined gyroscope with decoupled oscillation modes, Sens. Actuators A 97-98C (April 2002) 337-348.

[7] S.E. Alper, T. Akin, A symmetrical and decoupled nickel microgyroscope on insulating substrate, Sens. Actuators A 115 (September 2004) 336350 .

[8] S.E. Alper, T. Akin, A single-crystal silicon symmetrical and decoupled MEMS gyroscope on an insulating substrate, J. Microelectromech. Syst. 14 (August (4)) (2005) 707-717.

[9] S.E. Alper, K. Azgin, T. Akin, High-performance SOI-MEMS gyroscope with decoupled oscillation modes, in: Proc. 19th IEEE Int. Conf. Micro Electro Mechanical Systems (MEMS 2006), Istanbul, Turkey, 22-26 January, 2005, pp. 70-73.

[10] http://www.memsrus.com/nc-mumps.soi.html.

\section{Biographies}

Said Emre Alper was born in Ankara, Turkey, in 1976. He received the BS, $\mathrm{MSc}$, and $\mathrm{PhD}$ degrees in electrical and electronics engineering with high honors from Middle East Technical University (METU) in Ankara, in 1998, 2000, and 2005, respectively. Between 1998 and 2005, he has worked as a research assistant at METU in the Department of Electrical and Electronics Engineering, MEMSVLSI research group. Since 2006, he has been occupied as senior research scientist in the same department. His major research interests include capacitive inertial sensors, micromachined resonators and actuators, capacitive interface circuits, and microfabrication technologies. He is the first author of the symmetric and decoupled gyroscope design, which won the first prize award in the operational designs category of the "International Design Contest" organized by DATE and CMP in March 2001. He is also the first author of the tactical-grade symmetrical and decoupled micro-gyroscope design, which won the third prize award in the international "3-D MEMS Design Challenge" organized by MEMGEN Corporation (currently Microfabrica), in June 2003, among 132 MEMS designs from 24 countries and 25 states across United States. He received "METU Thesis of the Year Award" in 2000 and 2005, for his MSc thesis and PhD dissertation, respectively, awarded by Prof. Mustafa N. Parlar Education and Research Foundation.

Kivanc Azgin was born in Erzurum, Turkey, in 1982. He received his BS degree and Mechatronics Minor Certificate in Mechanical Engineering with honors from Middle East Technical University (METU) in Ankara, Turkey, in 2004 and 2005, respectively. Since 2004, he has been working as a research engineer at METU in the Department of Electrical and Electronics Engineering, MEMS VLSI Research Group. His major research interests include micromachined inertial sensors, resonating actuators, micro IC probes, micromechatronics and microfabrication technologies. He is now continuing his MS studies at METU in the Department of Electrical and Electronics Engineering.

Tayfun Akin was born in Van, Turkey, in 1966. He received the BS degree in electrical engineering with high honors from Middle East Technical University, Ankara, in 1987 and went to the USA in 1987 for his graduate studies with a graduate fellowship provided by NATO Science Scholarship Program through the Scientific and Technical Research Council of Turkey (TUBITAK). He received the MS degree in 1989 and the $\mathrm{PhD}$ degree in 1994 in electrical engineering, both from the University of Michigan, Ann Arbor. Since 1995, 1998, and 2004, he has been employed as an assistant professor, associate professor, and professor, respectively, in the Department of Electrical and Electronics Engineering at Middle East Technical University, Ankara, Turkey. $\mathrm{He}$ is also the technical coordinator of METU-MET, an IC fabrication factory which is transferred to Middle East Technical University by the government for MEMS related production. His research interests include micro-electromechanical systems (MEMS), microsystems technologies, infrared detectors and readout circuits, silicon-based integrated sensors and transducers, and ana$\log$ and digital integrated circuit design.He has served in various MEMS, EUROSENSORS, and TRANSDUCERS conferences as a Technical Program Committee member. He is the co-chair of The 19th IEEE International Conference of Micro Electro Mechanical Systems (MEMS 2006) held in Istanbul. 
$\mathrm{He}$ is the winner of the First Prize in Experienced Analog/Digital MixedSignal Design Category at the 1994 Student VLSI Circuit Design Contest organized and sponsored by Mentor Graphics, Texas Instruments, HewlettPackard, Sun Microsystems, and Electronic Design Magazine. He is the coauthor of the symmetric and decoupled gyroscope project which won the first prize award in the operational designs category of the international design contest organized by DATE Conference and CMP in March 2001. He is also the co-author of the gyroscope project which won the third prize award of 3 D MEMS Design Challenge organized by MEMGen Corporation (currently, Microfabrica). 